\title{
Biological role of phlorotannis extracted from brown algae and its activity in the diabetic treatment
}

\author{
Mai Abd-Elnaby \\ Chemistry Department, Faculty of Science, Tanta University, Egypt
}

\section{III}

Background: Diabetes mellitus is characterized by hyperglycemia, which is associated with failure of various organs. Aim: This study aimed to investigate biological activity including the antioxidant, antibacterial and antidiabetic activities of polyphenolic compound "phlorotannis" derived from Egyptian brown seaweed Cystoseira compressa and Sargassum linifolium. Materials and Methods: Phlorotannis were extracted and confirmed by different chemical and physical tests. The seaweed extracts exhibited higher antioxidant properties. Phlorotannis extracts showed antibacterial activity evaluated against species [Staphylococcus aureus, Escherichia coli, and Bacillus cereus]. In in vivo study, fifty white male albino rats were divided into six equal groups. The first three groups of rats are control normal, control C. compressa extract and control S. linifolium extract groups, the last three groups which are diabetic by intraperitoneal injection of streptozotocin had one diabetic control and diabetic that got $60 \mathrm{mg} / \mathrm{kg}$ of $C$. compressa phlorotannis extract and diabetic that got $60 \mathrm{mg} / \mathrm{kg}$ of $S$. linifolium after four weeks of diabetes induction. Results: Phlorotannis extracts decrease serum glucose, $\alpha$-amylase, glucosidase activity and liver malondialdehyde. However, serum insulin, hepatic glutathione and total antioxidant capacity "TAC" were significantly increased compared with diabetic non-treated groups. Also, phlorotannis make activation of AMPKa2 expression in skeletal muscle in treated group as compared to diabetic group. The histopathological results supported that phlorotannis extracts markedly reduce damage in $\beta$ cells of pancreas. Conclusion: This study confirmed that phlorotannis extract from $C$. compressa and S. linifolium algae have the capacity to act as antioxidant and antidiabetic agents. These results point phlorotannins extracts as potential pharmaceutical resources for human health.

Keywords: Antidiabetic; Antioxidant; Brown seaweed; Phlorotannis 